\title{
Revisión crítica de las fuentes de variabilidad en la medición de la prevalencia de esquizofrenia
}

\author{
Berta Moreno-Küstner, ${ }^{1}$ Carlos Martín, ${ }^{2}$ José Almenara ${ }^{3}$
}

Artículo original

\section{SUMMARY}

Epidemiologic research plays a very relevant role to know the prevalence of schizophrenia. However, sometimes data from different studies are compared without taking into account some methodological questions that influence the results. This paper reviews different methodological factors that influence variability of rates in schizophrenia prevalence. Also, we provide some generic recommendations for measuring prevalence. We have revised 52 studies which offer prevalence rates of schizophrenia. A big difference appears in the prevalence rates of schizophrenia which range between 1 to 45 per 1000 inhabitants. The factors founded can be summarized as follows: 1. type of prevalence; 2 . denominator population; 3. place of selection of cases; 4 . classification issue; 5 . diagnoses categories and 6. methods of diagnoses. In conclusion, in the epidemiological studies about schizophrenia prevalence we have to take into account the methodological factors involved in order to interprete and compare results from different studies.

Key words: Schizophrenia, prevalence studies, epidemiology.

\section{RESUMEN}

Conocer la prevalencia de la esquizofrenia y trastornos afines tiene una importancia relevante en la investigación epidemiológica y en la planificación de servicios. Sin embargo, existe una gran variabilidad en los resultados obtenidos en las diferentes investigaciones. El objetivo de este artículo es hacer una revisión crítica de los aspectos metodológicos de los estudios epidemiológicos que pueden influir en la medición de la prevalencia de esquizofrenia y trastornos afines y ofrecer una serie de recomendaciones generales para su medición. Se revisan 53 estudios epidemiológicos que relatan 76 tasas de prevalencia que oscilan entre 1 y 45 por 1000 habitantes. Se han encontrado seis factores metodológicos que creemos que están influyendo en la variabilidad de la medida de prevalencia de la esquizofrenia: 1. el tipo de prevalencia según el periodo de tiempo, siendo la más utilizada la prevalencia puntual; 2 . el rango de edad de la población de estudio, siendo lo más frecuente incluir a personas mayores de 18 años; 3. el ámbito de detección de los casos más frecuentemente utilizado es la población general; 4 . las clasificaciones de enfermedades utilizadas son la CIE y la DSM en similar proporción; 5. la categoría diagnóstica incluida frecuentemente en los estudios es el grupo de psicosis no afectivas; 6. el método de valoración diagnóstica más utilizado es la entrevista CIDI.

\section{Conclusión}

Consideramos que llegar a un consenso internacional para homogeneizar los aspectos metodológicos en los estudios epidemiológicos para calcular cifras de prevalencia de esquizofrenia nos facilitará la comparación de sus resultados.

Palabras clave: Esquizofrenia, estudios de prevalencia, epidemiología.

\section{INTRODUCCIÓN}

No hay duda de que la esquizofrenia es una enfermedad crónica y grave que supone una alta carga global. ${ }^{1,2}$ La investigación epidemiológica sobre la prevalencia de la esquizofrenia, a pesar de su larga historia, sigue suscitando un gran interés. Son numerosos los estudios realizados en distintas regiones del mundo y muy diferentes sus resultados. La tesis sobre una menor variabilidad en las tasas está avalada por los resultados del estudio internacional sobre esquizofrenia (International Study of Schizophrenia: ISoS) auspiciado por la $\mathrm{OMS}^{3}$ que, a partir de una metodología estandarizada, en-

Departamento de Personalidad, Evaluación y Tratamiento Psicológico. Facultad de Psicología. Universidad de Málaga. España.

Centro de Salud del Marquesado. Distrito Sanitario Granada Nordeste. España.

Departamento de Biomedicina, Biotecnología y Salud Pública. Universidad de Cádiz. España.

Correspondencia: Berta Moreno-Küstner. Departamento de Personalidad, Evaluación y Tratamiento Psicológico. Facultad de Psicología. Universidad de Málaga. Campus de Teatinos s/n, 29071 Málaga, España. E-mail: bertamk@uma.es

Recibido primera versión: 1o. de mayo de 2013. Segunda versión: 25 de noviembre de 2013. Aceptado: 2 de diciembre de 2013. 
contró cifras con una variabilidad de entre dos y tres veces. Una revisión de estudios publicados sobre prevalencia de la esquizofrenia muestra que la magnitud de dicha variabilidad podría oscilar entre dos y cinco veces. ${ }^{4}$ En la extensa revisión realizada por Saha et al. ${ }^{5}$ se incluyen 46 países y 188 estudios, el rango de la prevalencia de la esquizofrenia oscila entre cuatro y siete por 1000 personas. En este sentido, la ya clásica revisión de Torrey ${ }^{6}$ reporta una variación de 10 veces en la prevalencia de la esquizofrenia según las diferentes zonas de estudio, hallazgo compartido por Eaton. ${ }^{78}$ En definitiva la cuestión por resolver es si la esquizofrenia se puede considerar un trastorno que se distribuye de forma similar en diferentes partes del mundo. ${ }^{9}$

Un requisito imprescindible para la investigación epidemiológica es la posibilidad de contar con métodos válidos y consistentes para realizar estudios de investigación. ${ }^{10}$ Además de las causas etiológicas y ambientales, cada vez existe mayor consenso en considerar que la variabilidad de la prevalencia de la esquizofrenia también está influenciada por los aspectos metodológicos de los estudios. ${ }^{11}$

El objetivo de este artículo es hacer una revisión crítica de los aspectos metodológicos que pueden influir en la medición de la prevalencia de la esquizofrenia y los trastornos afines así como ofrecer una serie de recomendaciones generales para estandarizar los métodos para su medición.

\section{MÉTODO}

En esta revisión se han incluido estudios publicados a partir de 1990, en inglés o español, que ofrecen cifras de prevalencia de la esquizofrenia y los trastornos afines. Para identificar los estudios susceptibles de revisión se realizó una búsqueda bibliográfica usando los recursos electrónicos MEDLINE, PsychINFO, Web of Science y Scopus. También se revisó la bibliografía de los artículos principales y de las revisiones sistemáticas publicadas sobre el tema. Este proceso fue realizado por los autores BMK y CMG.

Se han excluido estudios realizados en poblaciones consideradas de riesgo (personas sin techo, presidiarios, etc.), los desarrollados en grupos poblacionales concretos en función de su edad (jóvenes, ancianos), género (mujeres, hombres), así como los basados en poblaciones ingresadas en instituciones psiquiátricas. También se han excluido los estudios centrados en trastornos psicóticos inducidos por sustancias, trastornos afectivos con rasgos psicóticos y trastornos psicóticos debidos a una condición médica, los cuales pueden presentar más variabilidad entre los diferentes países.

\section{RESULTADOS}

Se han analizado 52 artículos, correspondientes a 53 estudios (un estudio se incluye dos veces, pues se desarrolla en dos poblaciones diferentes), que comunican un total de 75 cifras de prevalencia de esquizofrenia y trastornos afines. Los artículos incluidos en esta revisión se han llevado a cabo en 23 países de todo el mundo, de manera que 28 estudios se han desarrollado en Europa, 11 en América, siete en Asia, cinco en Oceanía y, por último, dos estudios en África.

$\mathrm{El}$ anexo 1 incluye las referencias de todos los artículos reseñados en esta revisión. En el cuadro 1 se detallan los aspectos metodológicos de cada uno de los estudios. Finalmente, los artículos han sido clasificados de acuerdo a cada una de las características metodológicas utilizadas (cuadro 2).

A partir de esta revisión crítica, los aspectos que hemos encontrado que influyen más en la variabilidad de los resultados han sido los siguientes: 1) tipo de medida de prevalencia, según el periodo de estudio; 2) límite de edad inferior y superior de la población denominador; 3) ámbito de detección de los casos; 4) clasificación de enfermedades; 5) grupos de diagnósticos y 6) método de valoración diagnóstica.

A continuación se presenta la frecuencia con la que se utiliza cada factor en los diferentes estudios revisados y se describen cada uno de estos aspectos.

\section{Tipo de medida de prevalencia, según el periodo estudiado}

En los 53 estudios revisados (anexo 1) se presentan 76 cifras de prevalencia (en algunos estudios se calcula más de un tipo de medida) que oscilan entre uno y 45 por 1000 habitantes. La prevalencia puntual se ha calculado en el $40 \%$ de los estudios, la prevalencia anual se presenta en el $26 \%$ y la prevalencia vida en el 34\% (cuadros 1 y 2).

La prevalencia es una medida importante de morbilidad que se calcula como una proporción, dividiendo el número total de individuos que manifiestan la enfermedad entre el total de la población de referencia. Se pueden distinguir varios tipos de prevalencia, según el periodo de estudio: prevalencia puntual y prevalencia del periodo que puede ser anual o vida, las cuales pasamos a definir a continuación. ${ }^{12-14}$

\section{Prevalencia puntual}

Se define como la proporción de casos existentes (anteriores y nuevos) en una población en un único punto en el tiempo. Representa a los individuos que están enfermos en ese momento determinado. Se obtiene a partir de un estudio con un diseño transversal y no longitudinal. Debemos hacer referencia a la fecha donde se hizo el corte para obtener la información. Las mediciones realizadas en un intervalo de tiempo breve, por ejemplo una semana o un mes, se suelen denominar también prevalencias puntuales.

\footnotetext{
$N^{\circ}$ de casos existentes en una población $p=\frac{\text { definida en un momento o punto de tiempo }(t)}{N^{\circ} \text { total de personas en la población }}$
} 
Cuadro 1. Descripción de los estudios revisados según sus características metodológicas

\begin{tabular}{|c|c|c|c|c|c|c|c|c|}
\hline Autor y año (No. ref.) & Lugar & $\begin{array}{l}\text { Prevalencia } \\
1000 \text { hab. }\end{array}$ & $\begin{array}{c}2 \\
\operatorname{lnf}\end{array}$ & $\begin{array}{c}2 \\
\text { sup }\end{array}$ & 3 & 4 & 5 & 6 \\
\hline Arajärvi et al., 2005 (1) & Finlandia & Vida: 15,0 & 40 & 69 & Atendida & DSM-IV & 1 & Clínico - SCID \\
\hline Almeida et al., 1997 (2) & Brasil & $\begin{array}{l}\text { Puntual: } 9,3 \\
\text { Vida: } 12,0\end{array}$ & 15 & SL & Censal & DSM-III & 3 & SCID DSM-III \\
\hline Andrade et al., 2002 (3) & Brasil & $\begin{array}{l}\text { Puntual: 7,0 } \\
\text { Anual: } 8,0 \\
\text { Vida: } 19,0\end{array}$ & 18 & SL & Censal & CIE-10 & 3 & CIDI \\
\hline Andrews et al., 2001 (4) & Australia & $\begin{array}{l}\text { Puntual: } 4,0 \\
\text { Anual: } 4,0\end{array}$ & 18 & $\mathrm{SL}$ & Censal & CIE-10 + DSM-IV & 3 & CIDI \\
\hline Awas et al., 1999 (5) & Etiopia & $\begin{array}{l}\text { Puntual: 6,0 } \\
\text { Puntual: } 8,0\end{array}$ & 15 & 85 & Censal & CIE-10 & 1 & CIDI \\
\hline Bamrah et al., 1991 (6) & UK & $\begin{array}{l}\text { Puntual: 6,3 } \\
\text { Anual: } 7,0\end{array}$ & 15 & SL & Atendida & CIE-9 & 3 & PSE \\
\hline Bijl et al., 1998 (7) & Holanda & $\begin{array}{l}\text { Puntual: } 2,0 \\
\text { Anual: } 2,0 \\
\text { Vida: } 4,0\end{array}$ & 18 & 64 & Censal & DSM-III-R & 1 & CIDI \\
\hline Bourdon et al., 1992 (8) & EEUU & $\begin{array}{l}\text { Puntual: 7,0 } \\
\text { Anual: 10,0 } \\
\text { Vida: } 15,0\end{array}$ & 18 & SL & Censal & DSM-III & 2 & DIS \\
\hline Chen et al., 1993 (9) & Hong-Kong & Vida: 1,3 & 18 & 64 & Censal & DSM-III & 1 & DIS-III \\
\hline Chien et al., 2004 (10) & Taiwan & Anual: 4,4 & 18 & SL & Atendida & CIE-9 & 2 & Clínico \\
\hline Cho et al., 2007 (1 1) & Corea & $\begin{array}{l}\text { Anual: } 5,0 \\
\text { Vida: } 12,0\end{array}$ & 18 & 64 & Censal & DSM-IV & 3 & CIDI \\
\hline Cohidon et al., 2009 (12) & Francia & Vida: 27,0 & 18 & SL & Censal & CIE-10 & 4 & MINI \\
\hline Díaz-Martínez et al., 2003 13) & México & Puntual: 20,0 & 15 & 65 & Censal & CIE-10 & 3 & CIDI \\
\hline Harvey et al., 1996 (14) & UK & Puntual: 5,3 & 18 & SL & Atendida & DSM-III-R & 3 & MSP \\
\hline Herrera et al., 1990 (15) & España & Puntual: 10,7 & 18 & SL & Censal & CIE-8 & 3 & PSE \\
\hline Hosain et al., 2007 (16) & Bangladesh & Puntual: 11,7 & 18 & 60 & Censal & DSM-IV & 3 & Clínico \\
\hline Hovatta et al., 1997 (17 a) & Finlandia b & Vida: 12,1 & 35 & 54 & Atendida & DSM-III, CIE-8,CIE-9 & 4 & OPCRIT, CHECK LIST DSM III-R \\
\hline Hovatta et al., 1997 (17 b) & Finlandia a & Vida: 22,1 & 35 & 54 & Atendida & DSM-III, CIE-8,CIE-9 & 4 & OPCRIT, CHECK LIST DSM III-R \\
\hline Jablensky et al., 2000 (18) & Australia & Puntual: 4,7 & 18 & 64 & Atendida & CIE-10 & 4 & OPCRIT (DIP) \\
\hline Jacobi et al., 2004 (19) & Alemania & $\begin{array}{l}\text { Puntual: } 15 \\
\text { Anual: } 26,0 \\
\text { Vida: } 45,0\end{array}$ & 18 & 65 & Censal & DSM-IV & 4 & CIDI-M \\
\hline Jay et al., 1997 (20) & Francia & Anual: 14,9 & 15 & $\mathrm{SL}$ & Atendida & DSM-III-R & 3 & Clínico \\
\hline Jeffreys et al., 1997 (21) & UK & Puntual: 5,1 & 15 & 54 & Atendida & DSM-III-R & 3 & Clínico \\
\hline Jenkins et al., 1997 (22) & UK & Anual: 4,0 & 16 & 65 & Censal & CIE-10 & 3 & SCAN \\
\hline Jorgensen et al., 2013 (23) & Suecia & Anual: 6,7 & 18 & 64 & Atendida & CIE-10 & 2 & Clínico \\
\hline Kebede et al., 1999 (24) & Etiopía & $\begin{array}{l}\text { Puntual: } 3,0 \\
\text { Vida: } 9,0\end{array}$ & 15 & SL & Censal & CIE-10 & 2 & CIDI \\
\hline Kendler et al., 1996 (25) & EEUU & $\begin{array}{l}\text { Vida: } 13,0 \\
\text { Vida: } 22,0\end{array}$ & 15 & 54 & Censal & DSM-III-R & $\begin{array}{l}2 \\
4\end{array}$ & CIDI \\
\hline Kessler et al., 1994 (26) & EEUU & $\begin{array}{l}\text { Anual: } 5,0 \\
\text { Vida: } 7,0\end{array}$ & 15 & 54 & Censal & DSM-III-R & 3 & SCID-DSM III \\
\hline Kessler et al., 2005 (27) & EEUU & Anual: 3,0 & 18 & SL & Censal & DSM-IV & 3 & SCID \\
\hline Kringlen et al., 2001 (28) & Noruega & $\begin{array}{l}\text { Anual: } 2,0 \\
\text { Vida: } 4,0\end{array}$ & 18 & 65 & Censal & DSM-III-R & 3 & CIDI \\
\hline Lehtinen et al., 1990 (29) & Finlandia & Puntual: 13,0 & 30 & SL & Censal & CIE-9 & 1 & PSE \\
\hline Lora et al., 2007 (30) & Italia & Puntual: 2,4 & 14 & SL & Atendida & CIE-10 & 2 & Clínico \\
\hline McConnell et al., 2002 (31) & Irlanda & Anual: 4,3 & 18 & 65 & Censal & CIE-10 + DSM-IV & 1 & SCAN \\
\hline
\end{tabular}




\section{Cuadro 1. Continuación}

\begin{tabular}{|c|c|c|c|c|c|c|c|c|}
\hline McCreadie et al., 1997 (32) & $\begin{array}{l}\text { UK } \\
\text { Nithsdale } \\
\text { Nunhead } \\
\text { Noerwood }\end{array}$ & $\begin{array}{l}\text { Puntual: 2,4 } \\
\text { Puntual: 3,3 } \\
\text { Puntual: 1,9 }\end{array}$ & 18 & SL & Atendida & CIE-10 & 1 & OPCRIT \\
\hline $\begin{array}{l}\text { Myles-Worsley et al., } \\
1999 \text { (33) }\end{array}$ & Micronesia & Vida: 19,9 & 15 & SL & Atendida & DSM-III-R & 2 & SADS-L y clinico \\
\hline Ortega et al., 1995 (34) & España & Puntual: 1,3 & 15 & 65 & Censal & DSM-III-R & 1 & $\mathrm{CIS}$ \\
\hline Perala et al., 2000 (35) & Finlandia & Vida: 8,7 & 30 & $\mathrm{SL}$ & $\begin{array}{l}\text { Censal + } \\
\text { atendida }\end{array}$ & DSM-IV & 1 & SCID DSM-IV \\
\hline Phillips et al., 2009 (36) & China & Puntual: 7,8 & 18 & SL & Censal & DSM-IV & 1 & SCID DSM-IV \\
\hline Ran et al., 2003 (37) & China & Puntual: 4,1 & 15 & SL & Censal & $\mathrm{CIE}-10$ & 3 & PSE, SDSS \\
\hline Regier et al., 1993 (38) & EEUU & $\begin{array}{l}\text { Puntual: } 6,0 \\
\text { Anual: } 8,0 \\
\text { Vida: } 13,0\end{array}$ & 18 & SL & Censal & DSM-III & 1 & DIS \\
\hline Roca et al., 1999 (39) & España & Puntual: 5,0 & 15 & SL & Censal & CIE-10 & 2 & SCAN \\
\hline Ruggieri et al., 2000 (40) & Italia & Anual: 3,4 & 18 & SL & Atendida & CIE-10 & 3 & Clínico \\
\hline Scully et al., 1996 (41) & Irlanda & Vida: 3,9 & 18 & SL & Atendida & DSM-III-R & 1 & SCID DSM-III-R \\
\hline Seva A et al., 1992 (42) & España & Puntual: 2,5 & 15 & SL & Censal & DSM-III & 1 & $\mathrm{CIS}$ \\
\hline Shrout et al., 1992 (43) & Puerto Rico & $\begin{array}{l}\text { Vida: } 9,5 \\
\text { Vida: } 21,0\end{array}$ & 18 & 65 & Censal & DSM-III & 2 & DIS \\
\hline Thornicroft et al., 1998 (44) & UK & Anual: 5,2 & 15 & 85 & Atendida & CIE-10 & 3 & SCAN \\
\hline Tizon et al., 2006 (45) & España & Anual: 5,5 & 15 & SL & Atendida & DSM-IV & 1 & Clínico \\
\hline Van Os et al., 2001 (46) & Holanda & Vida: 3,7 & 18 & 64 & Censal & DSM-III-R & 3 & CIDI \\
\hline Vicente et al., 2004 (47) & Chile & Puntual: 1,0 & 15 & SL & Censal & DSM-III-R & 3 & CIDI \\
\hline Villaverde et al., 1993 (48) & España & Puntual: 6,5 & 15 & $\mathrm{SL}$ & Censal & DSM-III-R & 3 & $\mathrm{CIS}$ \\
\hline Waldo et al., 1999 (49) & Micronesia & Puntual: 6,8 & 15 & SL & Atendida & DSM-IV & 3 & SCIDP \\
\hline Widerlov et al., 1997 (50) & Suecia & Anual: 4,2 & 18 & $\mathrm{SL}$ & Atendida & DSM-III-R & 1 & Clínico \\
\hline Wittchen et al., 1992 (51) & Alemania & Vida: 7,2 & 18 & 55 & Censal & CIE-9 & 1 & DIS \\
\hline Xiang et al., 2008 (52) & China & Vida: 4,9 & 15 & SL & Censal & CIE-10 & 2 & CIDI \\
\hline Youssef et al., 1991 (53) & Irlanda & Anual: 3,3 & 15 & $\mathrm{SL}$ & Atendida & DSM-III-R & 1 & Clínico \\
\hline
\end{tabular}

Leyenda características metodológicas: 2: Límite edad población denominador; 3: Ámbito detección casos; 4: Clasificación enfermedades; 5: Categoría diagnóstica; 6: Método valoración diagnóstica.

Leyenda de categorías diagnosticas: 1. Sólo Esquizofrenia; 2. Esquizofrenia, t. esquizoafectivo, t. esquizofreniforme, t. ideas delirantes, t. psicótico breve en diferentes combinaciones; 3 . Trastornos psicóticos o psicosis no orgánicas; 4. Psicosis probable.

Leyenda método diagnóstico: CIDI: Composite International Diagnostic Interview; DIS: Diagnostic Interview Schedule; CIS: Clinical Interview Schedule; OPCRIT: Operational Criteria Checklist for Psychosis; SCID: Structure Clinical Interview for DSM-IV; SCAN: Schedules for Clinical Assessment in Neuropsychiatry; DIP: Diagnostic Interview for Psychoses; MSP: Manchester Scale Psychiatry; PSE: Present State Examination; SADS-L: Schedule for Affective Disorders and Schizophrenia; MINI: Mini-International Neuropsychiatric Interview.

En algunas ocasiones el denominador se define como población en riesgo de enfermar. Son las situaciones donde la población de referencia es un subconjunto de la población total.

Podemos dar la siguiente expresión para estimar la prevalencia: ${ }^{15}$

$$
P=\frac{C_{t}}{N_{t}},
$$

donde $C_{t}=N_{t}-N_{t}^{\prime}$ es el número de casos prevalentes en el tiempo $t$.

Así, si en un momento determinado $t$, tenemos 2271 casos de esquizofrenia en un área $\left(C_{t}\right)$ y el censo de población para la misma es de 225255 habitantes $\left(N_{t}\right)$, la prevalencia viene dada por:

$$
P=\frac{2271}{2271+222984}=0.01(1 \%)
$$

Suponiendo que la prevalencia fue obtenida mediante un muestreo aleatorio, podemos calcular el intervalo de confianza correspondiente. Dado que la prevalencia es una proporción, el intervalo de confianza (IC) vendrá dado por: ${ }^{16}$

$$
I C_{1-\alpha}(\pi)=\left(p \pm z_{\alpha / 2} \sqrt{\frac{p(1-p)}{n}}\right)
$$


Cuadro 2. Distribución de los artículos según aspectos metodológicos

\begin{tabular}{|c|c|c|}
\hline Aspectos metodológicos & Categorías & Número de referencia bibliográfica \\
\hline \multirow[t]{3}{*}{ 1. Tipos de prevalencia* } & Puntual & $2-8,13-16,18,19,21,24,29,30-32,34,36-39,42,47-50$ \\
\hline & Anual & $3,4,6-8,10,11,19,20,22,23,26-28,31,38,40,44,45,51,54$ \\
\hline & Vida & $1-3,7-9,11,12,17,19,23-26,28,33,35,38,41,43,46,52,53$ \\
\hline \multirow[t]{17}{*}{ 2. Límites de edad población de estudio } & Edad inferior & \\
\hline & 14 & 30 \\
\hline & 15 & $2,5,6,13,20,21,24-26,33,34,37,39,42,44,45,47,48,49,52,53$ \\
\hline & 16 & 22 \\
\hline & 18 & $\begin{array}{l}3,4,7,8,9,10,11,12,14,15,16,18,19,23,27,28,31,32,36,38,40,41,43, \\
46,50,51\end{array}$ \\
\hline & 30 & 29,35 \\
\hline & 35 & 17 \\
\hline & 40 & 1 \\
\hline & Edad superior & \\
\hline & 54 & $17,21,25,26$ \\
\hline & 55 & 52 \\
\hline & 60 & 16 \\
\hline & 64 & $7,9,11,18,23,46$ \\
\hline & 65 & $13,19,22,28,31,34,43$ \\
\hline & 69 & 1 \\
\hline & 85 & 5,44 \\
\hline & Sin Limite & $2,3,4,6,8,10,12,14,15,20,24,27,29,30,32,33,35-42,45,47-52,53$ \\
\hline \multirow[t]{2}{*}{ 3. Ámbitos de detección de casos } & Población General & $2-5,7-9,11-13,15,16,19,22,24-29,31,34-39,42,43,46-48,52$ \\
\hline & Población atendida & $1,6,10,14,17,18,20,21,23,30,32,33,40,41,44,45,49,50,53$ \\
\hline \multirow[t]{8}{*}{ 4. Clasificaciones de enfermedades } & $\mathrm{CIE}$ & \\
\hline & 8 & 15 \\
\hline & 9 & $6,10,51,29$ \\
\hline & 10 & $3,4,5,12,13,18,22,23,24,30,31,32,33,39,40,44,52$ \\
\hline & DSM & \\
\hline & III & $2,8,9,17,38,42,43$ \\
\hline & III-R & $7,14,20,21,25,26,28,33,34,41,46,47,48,50,53$ \\
\hline & IV & $1,11,16,19,27,35,36,45,49$ \\
\hline \multirow[t]{4}{*}{ 5. Categorías diagnósticas } & 1 & $1,5,7,9,29,31,32,34,35,36,38,41,42,45,50,51,53$ \\
\hline & 2 & $8,10,23,24,30,33,39,43,52$ \\
\hline & 3 & $2,3,4,6,11,13,14,15,16,20,21,22,26,27,28,37,40,44,46,47,48,49$ \\
\hline & 4 & $12,17,18,19,25$ \\
\hline \multirow[t]{8}{*}{ 6. Método de valoración diagnóstica } & CIDI & $3,4,5,7,11,13,19,24,25,26,46,47,52$ \\
\hline & PSE/SCAN & $6,15,22,29,31,37,39,44$ \\
\hline & DIS & $8,38,43,51,9$ \\
\hline & CLINICO & $10,16,20,21,23,30,40,45,50,53,1$ \\
\hline & CIS & $34,42,48$ \\
\hline & OPCRIT & $17,18,32$ \\
\hline & SCID & $2,26,27,35,36,41,49$ \\
\hline & OTROS & $12,14,33$ \\
\hline
\end{tabular}

*En cada estudio se puede presentar más de un tipo de prevalencia, por lo que se repite el número de referencia. Leyenda de las categorías diagnosticas: 1. Sólo Esquizofrenia; 2. Esquizofrenia, t. esquizoafectivo, t. esquizofreniforme, t. ideas delirantes, t.psicótico breve en diferentes combinaciones; 3 . Trastornos psicóticos o psicosis no orgánicas; 4 . Psicosis probable. 
donde $\pi$ representa la prevalencia poblacional, $p$ la prevalencia calculada para la muestra y $n$ el tamaño de muestra.

\section{Cálculo del intervalo de confianza}

Suponiendo que la prevalencia fue obtenida mediante un muestreo aleatorio, podemos calcular el intervalo de confianza correspondiente. Dado que la prevalencia es una proporción, el intervalo de confianza (IC) vendrá dado por: ${ }^{15}$

$$
I C_{1-\alpha}(\pi)=\left(p \pm z_{\alpha / 2} \sqrt{\frac{p(1-p)}{n}}\right)
$$

donde $\pi$ representa la prevalencia poblacional, $p$ la prevalencia calculada para la muestra y $n$ el tamaño de muestra.

\section{Prevalencia periodo (anual)}

Se han definido dos medidas más derivadas de la prevalencia puntual: ${ }^{15,17}$ la prevalencia periodo y la prevalencia vida o en el transcurso de la vida (lifetime prevalence).

La prevalencia de periodo se define como la razón de casos existentes (anteriores y nuevos) en una población en un periodo de tiempo. Habitualmente se refiere a un año, por lo que se denomina prevalencia anual.

$$
p p=\frac{\begin{array}{c}
N^{0} \text { de casos existentes en una población } \\
\text { definida en un momento } t_{0}+\text { casos nuevos entre } t_{0} y t
\end{array}}{\begin{array}{c}
N^{\circ} \text { total de personas en la población } \\
\text { a mitad del intervalo } t_{0}: t
\end{array}}
$$

Podemos estimar la prevalencia de período mediante la ecuación siguiente:

$$
P_{(t:: t)}=\frac{C_{t_{0}: t}}{N_{t_{0}: t}}
$$

donde $C_{(\mathrm{t} 0, \mathrm{t})}$ incluye tanto los casos prevalentes $\left(C_{0}\right)$ en $t_{0}$ como los incidentes $(I)$ detectados en el periodo definido $\left(t_{0}: t\right)$. Si el estudio se lleva a cabo en una cohorte fija, el denominador de la expresión puede ser reemplazado por $\left(N_{0}\right)$ el tamaño de la cohorte en el tiempo $t_{0}$.

Con la definición que hemos dado de prevalencia de período, puede expresarse la ecuación anterior en función de una medida de incidencia I, de tal forma que:

$$
\begin{gathered}
P_{\left(t_{0}: t\right)}=\frac{C_{t_{0}: t}}{N_{t_{0}: t}}=\frac{C_{0}+1}{N_{0}} \\
\text { Prevalencia periodo (vida) }
\end{gathered}
$$

Si el periodo de estudio se amplía a toda la vida del sujeto hablamos de prevalencia vida, la cual se define como la proporción de individuos en una población que han manifestado el trastorno en algún momento de su vida. Se incluyen no sólo aquellos individuos que al realizar la encuesta presentan el cuadro sino también los que lo han sufrido con anterioridad.

Para el cálculo de la prevalencia vida usamos la misma expresión que para estimar la prevalencia puntual $P$, donde
$C_{t}$ incluye ahora a las personas que tienen la enfermedad en el tiempo $t$, las personas que se curaron previamente de la enfermedad y aquéllas que están en un estado de remisión de la misma.

La cifra de prevalencia vida para un trastorno mental de larga evolución, como es la esquizofrenia, se asimila a la de riesgo vida, mientras que en enfermedades que pueden evolucionar hacia una mejoría la noción de riesgo no se puede equiparar a la prevalencia de vida. El riesgo vida es la probabilidad de que un trastorno o fenómeno particular apareciera si todos los individuos vivieran hasta una determinada edad. Como una forma de estimarla algunos autores calculan la proporción de sujetos en la población general que alguna vez han presentado el trastorno. Esto es la prevalencia vida.

A continuación se resumen algunas características de la prevalencia: $i$ ) es una proporción, que no será necesariamente un porcentaje ya que puede multiplicarse por un valor base diferente de 100 (1000, 10 000,...); ii) refleja acertadamente la magnitud de una enfermedad; iii) es útil para la gestión sanitaria, iv) depende de la frecuencia de aparición de casos nuevos y de la duración media de la enfermedad; v) es de poca utilidad en estudios etiológicos.

\section{Rango de edad de la población estudiada}

La mayoría de los estudios revisados en este trabajo delimitan la edad mínima de inclusión de los casos en 18 años (49\%), seguido, en segundo lugar (37\%), de estudios que determinan la edad mínima en 15 años. Por otra parte, la mayoría de los estudios (64\%) no indican límite superior de edad en la población a analizar.

\section{3. Ámbito de detección de los casos: población general o población atendida}

En los artículos revisados, la mayoría de los estudios (64\%) se han realizado en población general, mientras que el $36 \%$ se han llevado a cabo en personas atendidas tanto en servicios de salud mental como en los de atención primaria y en servicios sociales.

Si el estudio se realiza en la población general se obtienen cifras de prevalencia real, mientras que si los casos se seleccionan a partir de los servicios, en el caso nuestro concreto en uno de salud mental, serían tasas de prevalencia tratada, asistida o administrativa.

\section{En población general. \\ Estudios comunitarios (método censal)}

Los estudios comunitarios se pueden dividir en dos tipos: los estudios censales intensivos, que suelen realizarse en áreas geográficas muy reducidas (en este caso el estudio es muy exhaustivo y se entrevista a toda la población) y los estudios muestrales que incluyen, entre otros, los estudios en dos fases 
en los que se introduce una fase de cribado o screening antes de realizar la entrevista psiquiátrica ${ }^{18}$ (segunda fase).

$$
\begin{gathered}
\text { En población atendida (método informante clave } \\
\text { y registros de casos psiquiátricos) }
\end{gathered}
$$

Los estudios en la población atendida en servicios sanitarios (atención primaria y salud mental) y sociales, suelen utilizar el método del informante clave. Este procedimiento consiste en elaborar un listado de servicios e instituciones de un área determinada -normalmente pequeñas comunidades-, que pueden ser los lugares probables a los que acudan los posibles casos. Se deben seleccionar como informantes clave a personas que conozcan lo que ocurre en su medio.

Otra posibilidad es incluir solamente los casos que acuden a demandar atención a los servicios de salud mental de un área determinada (registro de casos psiquiátricos). Sin embargo, el número de casos se encuentra muy condicionado por el grado de desarrollo asistencial del lugar donde se realiza el estudio y, además, se perderán aquellos casos que no demanden atención.

\section{Cuestiones diagnósticas: clasificaciones de enfermedades, grupos diagnósticos y método de valoración diagnóstica}

La dificultad para conocer la causa exacta de la esquizofrenia y los trastornos afines, así como la carencia de clínica patognomónica o de pruebas diagnósticas fiables obligan a trabajar con un constructo difícil de definir, lo que complica su estudio epidemiológico. Teniendo en cuenta los problemas que encierra la definición de esquizofrenia, los estudios epidemiológicos deben ser interpretados bajo esta premisa. ${ }^{19}$ En la actualidad todavía se observa cómo tras décadas después de la introducción del concepto de esquizofrenia, la investigación sigue estando necesitada de una validez conceptual de este constructo, fundamentalmente de sus características psicopatológicas y de sus límites fenotípicos. ${ }^{20}$ Recientemente se están realizando estudios epidemiológicos en los que se analiza la psicosis desde una perspectiva dimensional. ${ }^{21}$

\section{Clasificación de enfermedades}

Respecto a las clasificaciones de enfermedades utilizadas en los 53 estudios revisados, la mayoría utiliza el DSM (Manual Diagnóstico y Estadístico de los Trastornos Mentales) $(58,5 \%)$, frente al resto que usa la CIE (Clasificación Internacional de Enfermedades) (cuadro 2). De todas maneras conviene destacar que en algunos estudios se utilizan ambas.

Los sistemas de clasificación de enfermedades tienen una importancia capital en la investigación epidemiológica, puesto que la aplicación de uno u otro puede afectar de forma considerable a los datos de prevalencia obtenidos en estudios de investigación, especialmente en el caso de la esquizofrenia. $^{22}$
Con el desarrollo de las distintas clasificaciones de enfermedades, tanto por parte de la Organización Mundial de la Salud, con la $\mathrm{CIE},{ }^{23}$ como de la Asociación Americana de Psiquiatría, con el DSM, ${ }^{24}$ se ha hecho un gran esfuerzo para basar en criterios operativos la clasificación de los trastornos mentales y utilizarlas de forma rutinaria en la práctica clínica y en la investigación. Si la CIE en su $9^{a}$ edición fue considerada por Kulhara et al. ${ }^{25}$ como un sistema útil y fiable para diagnosticar la esquizofrenia, el desarrollo de la décima versión de la CIE y la inclusión de criterios multiaxiales de la DSM-IV ha incrementado la posibilidad de comparar estos sistemas. Con la publicación reciente de la DSM- $5^{26}$ los criterios para el diagnóstico de la esquizofrenia y los trastornos afines no han cambiado sustancialmente. En concreto se han eliminado los tipos de esquizofrenia con el objetivo de crear mayor estabilidad diagnóstica. Ya que esta clasificación se ha publicado en 2013, todavía es pronto para cuantificar cómo ha influido en el cálculo de las cifras de prevalencia.

\section{Grupos diagnósticos}

En la mayoría (42\%) de los estudios epidemiológicos revisados se analiza el grupo de las psicosis no afectivas (esquizofrenia, trastornos esquizofreniformes, trastornos de ideas delirantes y psicosis atípicas) (código 3, cuadros 1 y 2). El siguiente diagnóstico más analizado $(32 \%)$ es la esquizofrenia -en este caso nos referimos exclusivamente a este trastorno independientemente- (código 1, cuadros 1 y 2).

Las diferentes categorías diagnósticas utilizadas por los investigadores en distintas partes del mundo y épocas han sido otra de las fuentes de variabilidad de las tasas de prevalencia de la esquizofrenia y los trastornos afines. Las primeras comparaciones entre tasas aportadas por estudios europeos y norteamericanos pusieron de manifiesto diferencias tan importantes que sólo podían explicarse por el manejo de una cultura diagnóstica distinta a uno y otro lado del Atlántico. ${ }^{22}$

\section{Método de valoración diagnóstica}

Existe gran diversidad en los métodos de valoración diagnóstica en los estudios epidemiológicos sobre la esquizofrenia y los trastornos afines. A partir de nuestra revisión hemos encontrado que los estudios analizados utilizan con más frecuencia el CIDI (Composite International Diagnostic Interview) (24.5\%), seguido de la entrevista clínica (20.7\%). Otros de los instrumentos utilizados son el PSE/SCAN (Present State Examination-Schedules for Clinical Assessment in Neuropsychiatry) (15.1\%), la SCID (Structure Clinical Interview for DSM-IV) (13.2\%), la DIS (Diagnostic Interview Schedule) (9.4\%).

Los denominados estudios epidemiológicos de tercera generación buscan reducir el peso de esta fuente de variabilidad, dando un salto cualitativo y cuantitativo, fundamen- 
talmente con la introducción de instrumentos diagnósticos estructurados. Estos métodos normalizados representan un importante paso para alcanzar una mayor fiabilidad en la investigación epidemiológica. Además, la mayoría de estos instrumentos están traducidos y validados en numerosos idiomas, lo que permite utilizarlos en estudios internacionales.

El primer instrumento desarrollado para determinar el diagnóstico de manera estructurada y sistemática fue el PSE $^{27}$ que evolucionó y mejoró hasta producir el PSE-9 y el PSE-10, según si permiten usar criterios de clasificación de la CIE-9 o de la CIE-10, respectivamente. La última versión de este instrumento es la entrevista clínica semi-estructurada SCAN. ${ }^{28}$ Están diseñados para ser utilizados en el ámbito clínico y se necesita experiencia en esta área para su aplicación, además de un entrenamiento específico.

En 1980 se desarrolló la Pauta de Entrevista Diagnóstica-DIS ${ }^{29}$ para su uso en estudios epidemiológicos en población general. La Entrevista Compuesta Diagnóstica Internacional-CIDI, de posterior desarrollo, ${ }^{30}$ está basada en la DIS. Es una entrevista muy estructurada y fácil de utilizar, para la que no es necesario tener experiencia clínica, aunque sí se precisa un entrenamiento reglado.

Otro instrumento apropiado para la detección de trastornos graves como la esquizofrenia es la Entrevista Clínica Estructurada - SCID-II, ${ }^{31}$ que debe ser aplicada por personas legas entrenadas en el manejo de la entrevista.

\section{DISCUSION}

\section{Limitaciones}

En primer lugar nos gustaría indicar que somos conscientes de las limitaciones de este estudio, las cuales se enumeran a continuación. Si bien el uso de revisiones sistemáticas ha ofrecido una nueva perspectiva al panorama de la epidemiología de la esquizofrenia, ${ }^{32}$ este artículo no pretende ser un meta-análisis pues no se ha seguido la metodología necesaria para ello, ni se incluyen todos los artículos publicados sobre el tema. Otra limitación de este trabajo es que se centra solamente en algunos aspectos metodológicos, aunque existen otros factores que también pueden influir en las cifras de prevalencia como, por ejemplo, el tamaño de la muestra del estudio, el tipo de muestreo para seleccionar los casos, el cálculo del error estándar y que no hemos incluido en esta revisión. A pesar de estas limitaciones consideramos que se trata de una revisión crítica que aporta información relevante en el ámbito de la epidemiología psiquiátrica.

\section{Principales resultados y recomendaciones}

La principal aportación de esta revisión es que analizamos los aspectos metodológicos más utilizados en 53 estudios epidemiológicos los cuales suponen una amplia represen- tación de la investigación epidemiológica reciente sobre esquizofrenia (anexo 1, cuadro 1).

Se han encontrado seis factores metodológicos que creemos que están influyendo en la variabilidad de la medida de prevalencia de la esquizofrenia: 1) el tipo de prevalencia según el periodo de tiempo (puntual, anual y vida), siendo la más utilizada la prevalencia puntual; 2) el rango de edad de la población de estudio es también muy variable, siendo lo más frecuente incluir a personas mayores de 18 años y sin límite de edad superior; 3) el ámbito de detección de los casos varía (población general y atendida en servicios sanitarios -atención primaria y de salud mental- y sociales) siendo los más frecuentes estudios realizados en la población general; 4) las clasificaciones de enfermedades utilizadas son la CIE y la DSM en una proporción similar; 5) la categoría diagnóstica incluida más frecuentemente en los estudios es el grupo de psicosis no afectivas y 6) el método de valoración diagnóstica más utilizado es la entrevista CIDI, seguida del diagnóstico clínico, aunque hemos encontrado más de ocho métodos de valoración diagnóstica (cuadro 2).

A continuación ofrecemos algunas recomendaciones que consideramos pueden ser útiles para llevar a cabo estudios epidemiológicos sobre la prevalencia de la esquizofrenia, así como los sesgos que se pueden presentar en los diferentes factores analizados.

\section{Tipo de medida de prevalencia según el periodo estudiado: puntual y periodo (anual y vida)}

En los estudios sobre esquizofrenia, la prevalencia puntual es un término más conceptual que real puesto que, en la práctica, resulta difícil recabar la información de los casos en un instante determinado. Cuando de enfermedades de larga evolución se trata, más aún cuando éstas se presentan de forma insidiosa como ocurre en la esquizofrenia, resulta aconsejable que el periodo contemplado abarque como mínimo seis meses o un año.

\section{Rango de edad de la población estudiada}

Debido a que los trastornos esquizofrénicos no se inician habitualmente en la infancia, para el cálculo de las tasas se debe usar como denominador la población mayor de 15 o 18 años ya que incluir personas por debajo de esa edad disminuiría las tasas de forma artificial. En algunos casos se suele determinar también un límite superior de edad, por ejemplo un máximo de 55 años, circunstancia que conviene tener en cuenta cuando se comparan tasas de prevalencia de la esquizofrenia y los trastornos afines.

\section{3. Ámbito de detección de los casos: población general y atendida}

En los estudios epidemiológicos, la detección de los casos se realiza habitualmente en la población general. Sin embargo, la baja frecuencia de la esquizofrenia hace que este tipo de estudios sea poco apropiado para el cálculo de tasas de prevalencia 
porque se necesitaría entrevistar a una población muy amplia para conseguir recabar un número de casos estadísticamente representativo. Además, estos estudios pueden presentar un sesgo de selección ya que un grupo importante de personas con trastornos mentales graves pueden estar residiendo en instituciones o alojamientos protegidos y es difícil acceder a este grupo de población. ${ }^{33}$ En el estudio NEMESIS ${ }^{34}$ se calculó una pérdida de $0.05 \%$ de los casos por este motivo. Otro posible sesgo de selección se presentaría por la dificultad de incluir a personas sin techo. ${ }^{34}$ Por último, las personas con esquizofrenia y trastornos afines no son muy proclives a contestar encuestas extensas ${ }^{11}$ por lo que se puede dar un sesgo de información.

Cuando queremos obtener cifras de prevalencia de la esquizofrenia es más efectivo realizar los estudios en la población atendida en el ámbito socio-sanitario (servicios de salud mental y atención primaria). En el caso de la esquizofrenia es un buen diseño, dado que quienes presentan la enfermedad suelen acudir a lo largo de su vida a algún tipo de servicio sanitario o social. Actualmente no sería pertinente realizar estudios epidemiológicos sobre la esquizofrenia solamente en el ámbito hospitalario pues se presentaría el sesgo de Berkson y, por otro lado, a partir de la reforma psiquiátrica los pacientes con esquizofrenia acuden a una gran variedad de servicios, tanto sanitarios como sociales, para buscar atención. No hay que olvidar que estas cifras siempre se refieren a las personas tratadas o atendidas. ${ }^{35-37}$ Para determinar la verdadera dimensión de esta enfermedad, como concluyeron Përalä et al., ${ }^{11}$ habría que utilizar el mayor número de fuentes de información posibles.

\section{Cuestiones diagnósticas}

\section{Clasificaciones de enfermedades, 5. Grupos diagnósticos y \\ 6. Método de valoración diagnóstica}

Los problemas derivados especialmente de la falta de normalización de los criterios diagnósticos impiden, de manera significativa, la comparación entre los resultados dados a conocer por los investigadores y obtenidos en distintas culturas. Las diferencias y los desacuerdos que afectan a los estándares diagnósticos se basan en el empleo de criterios estrechos frente a criterios amplios, ${ }^{38}$ es decir, en la dicotomía entre especificidad (grado de detección de los verdaderos negativos) y sensibilidad (grado de detección de los verdaderos positivos).

Para diagnosticar casos de esquizofrenia lo más adecuado es la utilización de entrevistas clínicas estructuradas, aunque no siempre es posible su utilización en estudios epidemiológicos por las exigencias en su entrenamiento.

Como se ha demostrado en el estudio ECA -Epidemiologic Catchment Area- ${ }^{39}$ la valía de la entrevista DIS para cuantificar la esquizofrenia en muestras de población general es pequeña, debido a que detecta muchos falsos positivos. También, el módulo de esquizofrenia de la entrevista CIDI, uno de los instrumentos más utilizados en estudios poblacionales, genera falsos positivos en estudios censales. ${ }^{40}$ En ambos casos podría presentarse un sesgo de selección por el uso inapropiado de pruebas diagnósticas que puede inducir a incluir pacientes con diferentes criterios diagnósticos. También puede darse un sesgo de información en los estudios epidemiológicos sobre esquizofrenia, ya que para realizar el diagnóstico necesitamos recabar información del propio sujeto y, en algunas ocasiones, no son proclives a facilitar dicha información. La mejora en la fiabilidad de los diagnósticos ha influido en las cifras de prevalencia en los estudios epidemiológicos. ${ }^{41}$

\section{CONCLUSIÓN}

La principal conclusión de este trabajo es que existe una gran heterogeneidad en los aspectos metodológicos de los estudios analizados y que todo ello está influyendo en las cifras obtenidas.

Creemos que sería de gran utilidad llegar a un consenso internacional para utilizar criterios estandarizados que nos permitan comparar, de forma fiable, los resultados de los estudios epidemiológicos, en general, y sobre la esquizofrenia y los trastornos afines, en particular.

La homologación y estandarización de estos procedimientos determinará definitivamente si realmente existen diferencias en los distintos lugares del mundo sobre la prevalencia de la esquizofrenia o si estas diferencias se deben a factores puramente metodológicos. Si aplicamos de forma homogénea estos aspectos metodológicos podremos ofrecer información más fiable al complejo paisaje de la epidemiología de la esquizofrenia.

\section{AGRADECIMIENTOS}

A la Junta de Andalucía por financiar los siguientes proyectos de investigación de la Consejería de Sanidad (PI338/2008 y PI332/2008) y de la Consejería de Innovación, Ciencia y Empresa (P10-CTS-5862) y por financiar el grupo GAP (CTS-945). A la Asociación Científica PSICOST.

\section{REFERENCIAS}

1. Murray CJ, Lopez AD (eds). The global burden of disease: A comprehensive assessment of mortality and disability from diseases, injuries, and risk factors in 1990 and projected to 2020. Boston: Harvard School of Public Health; 1996.

2. Catalá-López F, Gènova-Maleras R, Álvarez-Martín E, Fernández de Larrea-Baz $\mathbf{N}$ et al. Carga de enfermedad en adolescentes y jóvenes en España. Rev Psiquiatr Salud Ment 2013;06:80-5.

3. Jablensky A, Sartorius N, Ernberg G, Anker M et al. Schizophrenia: manifestations, incidence and course in different cultures. A world health organization ten-country study. Psychol Med 1992;20 (monog. supp.).

4. Goldner E, Hsu L, Waraich P, Somers J. Prevalence and incidence studies of schizophrenic disorders: a systematic review of the literature. Can J Psychiatry 2002;47(9):833-843.

5. Saha S, Chant D, Welham J, McGrath J. A systematic review of the prevalence of schizophrenia. PLoS Med 2005;2 (5):413-433.

6. Torrey EF. Prevalence studies in schizophrenia. Br J Psychiatry 1987;150:598-608. 
7. Eaton WW. Epidemiology of schizophrenia. Epidemiol Rev 1985;7:105126.

8. Eaton WW. Updata on the epidemiology of schizophrenia. Epidemiol Rev 1991;13:320-328.

9. McGrath JJ. The surprisingly rich contours of schizophrenia epidemiology. Arch Gen Psychiatry 2007;64:14-16.

10. Fletcher RH, Fletcher SW, Wagner EH. Epidemiología clínica. Aspectos fundamentales. Barcelona: Masson, 2002.

11. Përalä J, Suvisaari J, Saarni SI, Kuoppasalmi K et al. Lifetime prevalence of psychotic and bipolar I disorders in a general population. Arch Gen Psychiatry 2007;64:19-28.

12. MacMahon B, Pugh TF. Epidemiology: Principles and methods. Boston: Little, Brown; 1970.

13. Mausner JS, Bahn AK. Epidemiology: An introductory text. Philadelphia: Philadelphia Saunders; 1974.

14. Bolúmar F. Las medidas de frecuencia. En: Doménech (ed.). Diseño de estudios sanitarios. Estudios descriptivos. Barcelona: UD 2 Signo; 2000.

15. Kleinbaum DG, Kupper LL, Morgenstern H. Epidemiologic research: principles and quantitative methods. New York: Van Nostrand Reinhold; 1982.

16. Bolúmar F. Medición de los fenómenos de salud y enfermedad en epidemiología. En: Piédrola Gil (ed.). Medicina preventiva y salud pública. Décima edición. Barcelona: Masson; 2001.

17. Rothman JK, Greenland S. Modern epidemiology. Segunda edición. Philadelphia: Lippincott Williams \& Wilkins; 1998.

18. Vázquez Barquero JL, Diez Marnique JF, Peña $C$, Aldama J et al. A community mental health survey in Cantabria: a general description of morbidity. Psychol Med 1987;17:227-241.

19. Bhugra D. The global prevalence of schizophrenia. PLoS Med 2005;2(5):372-373.

20. Jansson LB, Parnas J. Competing definitions of schizophrenia: What can be learned from polydiagnostic studies? Schizophr Bull 2007;33(5):1178-1200.

21. Os J van, Hanssen M, Bijl Rv, Vollebergh W. Prevalence of psychotic disorder and community level of psychotic symptoms: an urban-rural comparison. Arch Gen Psychiatry 2001;58:663-668.

22. Warner R, Girolamo G. Esquizofrenia. Epidemiología de los trastornos mentales y de los problemas psicosociales. Ginebra: Meditor; 1995.

23. Organización Mundial de la Salud. Clasificación Internacional de Enfermedades. $10^{\mathrm{a}}$ revisión. Ginebra: 1992.

24. López Ibor JM. Manual diagnóstico y estadístico de los trastornos mentales: DSM-IV-TR. American Psychiatric Association. Barcelona: Masson; 2000.

25. Kulhara P, Mattoo SK, Chandiramani K, Bhave S et al. Diagnostic systems for schizophrenia. A cross-sectional study concordance from
India. Acta Psychiatr Scand 1986;74:55-61.

26. American Psychiatric Association. Diagnostic and Statistical Manual of Mental Disorders (DSM-5), $5^{\text {a }}$ edición. Arlington VA: 2013.

27. Wing JK, Cooper JE, Sartorius N. The description and manual for the PSE and CATEGO system. Cambridge: Cambridge University Press; 1974.

28. Wing JK, Babor T, Brugha Ts, Burke $H$ et al. SCAN: Schedules for clinical assessment in neuropsychiatry. Arch Gen Psychiatry 1990;47:489-593.

29. Robins LN, Helzer JN, Crougham J, Ratcliff KS. National Institute of Mental Health Diagnostic Interview Schedule. Its history, characteristics, and validity. Arch Gen Psychiatry 1981,38:381-389.

30. Robins LN, Wing J, Wittchen HU, Helzer JE et al. The composite international diagnostic interview: An epidemiologic instrument suitable for use in conjunction with different diagnostic systems and in different cultures. Arch Gen Psychiatry 1988;45:1069-1077.

31. First MB, Gibbon M, Spitzer RL et al. User's guide for the structured clinical interview for DSM-IV. Axis II personality disorders (SCID-II). Washington DC: American Psychiatric Press; 1997.

32. Saha $S$, Chant $D$, Mcgrath J. Meta-analyses of the incidence and prevalence of schizophrenia: conceptual and methodological issues. Int J Methods Psychiatr Res 2008;17(1):55-61.

33. Chien I, Chou Y, Lin C, Bih S et al. Prevalence of psychiatric disorders among National Health Insurance enrollees in Taiwan. Psychiatr Serv 2004;55:691-697.

34. Bijl RV, Ravelli A, Van Zessen G. Prevalence of psychiatric disorder in the general population: results of The Netherlands Mental Health Survey and Incidence Study (NEMESIS). Soc Psychiatry Psychiatr Epidemiol 1998;33:587-595.

35. Häfner H, Heiden W. an der. The contribution of European case registers to research on schizophrenia. Schizophr Bull 1986;12:26-49.

36. Freeman $\mathrm{H}$, Henderson JH. Evaluation of comprehensive care of the mentally ill. Londres: Gaskell; 1991.

37. Torres González F, Rosales Varo C, Moreno Küstner B. Prevalencia de la esquizofrenia. Monografías Psiquiatría 2002;2:2-6.

38. Sartorius N, Jablensky A, Ernberg G, Leff J et al. Course of schizophrenia in different countries: some results of a WHO international comparative 5-year follow-up study. En: Häfner H, Gattaz WF, Janzarik W (eds). Search for the causes of schizophrenia. Berlin: Springer-Verlag: 1987.

39. Keith SJ. Schizophrenic disorders in America. En: Robins L, Regier DA (eds.). Psychiatric disorders in America. Nueva York: Free Press; 1991; p. 33-51.

40. Andrews G, Henderson S, Hall W. Prevalence, comorbidity, disability and service utilisation. Overview of the Australian National Mental Health Survey. Br J Psychiatry 2001;178:145-153.

41. Moreno-Altamirano A, López-Moreno S, Corcho-Berdugo A. Principale medidas en epidemiologia. Salud Pública México 2000;42:337-348.

Artículo sin conflicto de intereses 
Fuentes de variabilidad en la medición de la prevalencia de esquizofrenia

ANEXO 1. Estudios epidemiológicos publicados desde 1990 que ofrecen cifras de prevalencia de la esquizofrenia. Estudios incluidos en nuestra revisión

1. Arajärvi R, Suvisaari J, Suokas J, Schreck M, Haukka J, Hintikka J, et al. Prevalence and diagnosis of schizophrenia based on register, case record and interview data in an isolated Finnish birth cohort born 1940-1969. Soc Psychiatry Psychiatr Epidemiol. 2005 Oct; $40(10): 808-816$.

2. Almeida-Filho N, Mari JDJ, Coutinho E, França JF, Fernandes J, Andreoli SB, et al. Brazilian multicentric study of psychiatric morbidity. Methodological features and prevalence estimates. Br J Psychiatry. 1997 Dic;171:524-529.

3. Andrade L, Walters EE, Gentil V, Laurenti R. Prevalence of ICD-10 mental disorders in a catchment area in the city of São Paulo, Brazil. Soc Psychiatry Psychiatr Epidemiol. 2002 Jul;37(7):316-25.

4. Andrews G, Henderson S, Hall W. Prevalence, comorbidity, disability and service utilisation. Overview of the Australian National Mental Health Survey. Br J Psychiatry. 2001 Feb; 178:145-153.

5. Awas M, Kebede D, Alem A. Major mental disorders in Butajira, southern Ethiopia. Acta Psychiatr Scand Suppl. 1999;397:56-64.

6. Bamrah JS, Freeman HL, Goldberg DP. Epidemiology of schizophrenia in Salford, 1974-84. Changes in an urban community over ten years. Br J Psychiatry. 1991 Dic;159:802-810.

7. Bijl RV, Ravelli A, van Zessen G. Prevalence of psychiatric disorder in the general population: results of The Netherlands Mental Health Survey and Incidence Study (NEMESIS). Soc Psychiatry Psychiatr Epidemiol. 1998 Dic;33(12):587-595.

8. Bourdon KH, Rae DS, Locke BZ, Narrow WE, Regier DA. Estimating the prevalence of mental disorders in U.S. adults from the Epidemiologic Catchment Area Survey. Public Health Rep. 1992 Dic;107(6):663-668.

9. Chen CN, Wong J, Lee N, Chan-Ho MW, Lau JT, Fung M. The Shatin community mental health survey in Hong Kong. II. Major findings. Arch Gen Psychiatry 1993, 50:125-133.

10. Chien I, Chou Y, Lin C, Bih S, Chou P. Prevalence of psychiatric disorders among National Health Insurance enrollees in Taiwan. Psychiatr Serv. 2004 Jun;55(6):691-697.

11. Cho M, Kim J, Jeon HJ, Suh T, Chung I, Hong JP, et al. Lifetime and 12-month prevalence of DSM-IV psychiatric disorders among Korean adults. J. Nerv. Ment. Dis. 2007 Mar; 195(3):203-210.

12. Cohidon C, Imbernon E, Gorldberg M. Prevalence of common mental disorders and their work consequences in France, according to occupational category. Am. J. Ind. Med. 2009 Feb;52(2):141-152.

13. Díaz-Martínez A, Díaz-Martínez R, Osornio-Rojo A, Rascón-Gasca ML. [Mental health in a municipality of the State of Querétaro: a community-based psychiatric research model]. Gac Med Mex. 2003 Abr;139(2):101-107.

14. Harvey CA, Pantelis C, Taylor J, McCabe PJ, Lefevre K, Campbell PG, et al. The Camden schizophrenia surveys. II. High prevalence of schizophrenia in an inner London borough and its relationship to socio-demographic factors. Br J Psychiatry. 1996 Abr;168(4):418-426.

15. Herrera R, Autonell J, Spagnolo E, Gispert R. Estudio epidemiológico en salud mental del al comarca del Baix Llobregat (Barcelona). Inf Psiquiatricas 1990;120: $111-130$.

16. Hosain GMM, Chatteriee N, Ara N, Islam T. Prevalence, pattern and determinants of mental disorders in rural Bangladesh. Public Health. 2007 Ene; $121(1): 18-24$.

17. Hovatta I, Terwilliger JD, Lichtermann D, Mäkikyrö T, Suvisaari J, Peltonen L, et al. Schizophrenia in the genetic isolate of Finland. Am. J. Med. Genet. 1997 Jul 25;74(4):353-360.

18. Jablensky A: Prevalence and incidence of schizophrenia spectrum disorders: implications for prevention. Aust N Z J Psychiatry 2000,34 Suppl:S26-34; discussion S35-28.

19. Jacobi F, Wittchen H, Holting C, Höfler M, Pfister H, Müller N, et al. Prevalence, co-morbidity and correlates of mental disorders in the general population: results from the German Health Interview and Examination Survey (GHS). Psychol Med. 2004 May;34(4):597-61 1.

20. Jay M, Gorwood P, Feingold J, Leboyer M: A one year prevalence study of schizophrenia on Reunion Island. Eur Psychiatry 1997, 12:284-288.

21. Jeffreys SE, Harvey CA, McNaught AS, Quayle AS, King MB, Bird AS. The Hampstead Schizophrenia Survey 1991. I: Prevalence and service use comparisons in an inner London health authority, 1986-1991. Br J Psychiatry. 1997 Abr; 170:301-306.

22. Jenkins R, Lewis $G$, Bebbington $P$, Brugha $T$, Farrell M, Gill B, et al. The National Psychiatric Morbidity surveys of Great Britain-initial findings from the household survey. Psychol Med. 1997 Jul;27(4):775-789.

23. Jörgensen I, Dalman C, Allebeck P. Prevalence of psychoses in Stockholm County. A population-based study using comprehensive healthcare registers. Nord J Psychiatry 2013; 00: 1-6.

24. Kebede D, Alem A: Major mental disorders in Addis Ababa, Ethiopia. I. Schizophrenia, schizoaffective and cognitive disorders. Acta Psychiatr Scand Suppl 1999, 397:1 1-17.

25. Kendler KS, Gallagher TJ, Abelson JM, Kessler RC. Lifetime prevalence, demographic risk factors, and diagnostic validity of nonaffective psychosis as assessed in a US community sample. The National Comorbidity Survey. Arch. Gen. Psychiatry. 1996 Nov;53(1 1): $1022-1031$.

26. Kessler RC, McGonagle KA, Zhao S, Nelson CB, Hughes M, Eshleman S, et al. Lifetime and 12-month prevalence of DSM-III-R psychiatric disorders in the United States. Results from the National Comorbidity Survey. Arch. Gen. Psychiatry. 1994 Ene;51 (1):8-19. 
ANEXO 1. Continuación

27. Kessler RC, Chiu WT, Demler O, Merikangas KR, Walters EE. Prevalence, severity, and comorbidity of 12-month DSM-IV disorders in the National Comorbidity Survey Replication. Arch. Gen. Psychiatry. 2005 Jun;62(6):617-627.

28. Kringlen E, Torgersen S, Cramer V. A Norwegian psychiatric epidemiological study. Am J Psychiatry. 2001 Jul;158(7):1091-1098.

29. Lehtinen $V$, Joukamaa M, Lahtela K, Raitasalo R, Jyrkinen E, Maatela J, et al. Prevalence of mental disorders among adults in Finland: basic results from the Mini Finland Health Survey. Acta Psychiatr Scand. 1990 May;81 (5):41 8-425.

30. Lora A, Bezzi R, Erlicher A. Estimating the Prevalence of Severe Mental Illness in Mental Health Services in Lombardy (ltaly). Com Mental Health J, 2007 August 43(4):341-356.

31. McConnell P, Bebbington P, McClelland R, Gillespie K, Houghton S. Prevalence of psychiatric disorder and the need for psychiatric care in Northern Ireland. Population study in the District of Derry. Br J Psychiatry. 2002 Sep;181:214-219.

32. McCreadie RG, Leese M, Tilak-Singh D, Loftus L, MacEwan T, Thornicroft G: Nithsdale, Nunhead and Norwood: similarities and differences in prevalence of schizophrenia and utilisation of services in rural and urban areas. Br J Psychiatry 1997, 170:31-36.

33. Myles-Worsley M, Coon H, Tiobech J, Collier J, Dale P, Wender P, Reimherr F, Polloi A, Byerley W: Genetic epidemiological study of schizophrenia in Palau, Micronesia: prevalence and familiality. Am J Med Genet 1999, 88:4-10.

34. Ortega MA, Seva A, perez A. Morbilidad psiquica diagnostica en la población general de La Rioja. An. Psiquiatria. 1995;2(9):320-326.

35. Perälä J, Suvisaari J, Saarni SI, Kuoppasalmi K, Isometsä E, Pirkola S, et al. Lifetime prevalence of psychotic and bipolar I disorders in a general population. Arch. Gen. Psychiatry. 2007 Ene;64(1):19-28.

36. Phillips MR, Zhang J, Shi Q, Song Z, Ding Z, Pang S, et al. Prevalence, treatment, and associated disability of mental disorders in four provinces in China during 2001-05: an epidemiological survey. Lancet. 2009 Jun 13;373(9680):2041-2053.

37. Ran M, Xiang M, Li SX, Shan Y, Huang M, Li SG, Liu Z, Chen E, Chan C: Prevalence and course of schizophrenia in a Chinese rural area. Aust N Z J Psychiatry 2003; 37:452-457.

38. Regier DA, Farmer ME, Rae DS, Myers JK, Kramer M, Robins LN, George LK, Karno M, Locke BZ. One-month prevalence of mental disorders in the United States and sociodemographic characteristics: the Epidemiologic Catchment Area study. Acta Psychiatr Scand 1993, 88:35-47.

39. Roca M, Gili M, Ferrer V, Bernardo M, Montaño JJ, Salvà JJ, et al. Mental disorders on the island of Formentera: prevalence in general population using the Schedules for Clinical Assessment in Neuropsychiatry (SCAN). Soc Psychiatry Psychiatr Epidemiol. 1999 Ago;34(8):410-415.

40. Ruggeri $M$, Leese $M$, Thornicroft G, Bisoffi G, Tansella M. Definition and prevalence of severe and persistent mental illness. Br J Psychiatry. 2000;177:149-155.

41. Scully P, Owens J, Kinsella A, Waddington JL. Schizophrenia, schizoaffective and bipolar disorder within an epidemiologically complete, homogeneous population in rural Ireland: small area variation in rate. Schizophrenia Research 67 (2004) 143- 155.

42. Seva A, Magallon R,Sarasola A, Merino JA. Investigacion Epidemiologica psiquiatrica en dos fases en la ciudad de Zaragoza. (El proyecto SAMAR-89). An. Psiquiatria. 1992;8(2):45-55.

43. Shrout PE, Canino GJ, Bird HR, Rubio-Stipec M, Bravo M, Burnam MA: Mental health status among Puerto Ricans, Mexican Americans, and non-Hispanic whites. Am J Community Psychol 1992, 20:729-752.

44. Thornicroft g, Strathdee G, Phelan M, Holloway F, Wykes T, Dunn G, et al. Rationale and design. PRiSM Psychosis Study I. Br j Psychiatry $1998 ; 173(11): 363-370$.

45. Tizón J, Ferrando J, Parés A, Artigué J, Parra B, Pérez C. Schizophrenic disorders in primary care mental health. Aten Primaria. 2007;39(3) 1 19-26.

46. Van Os J, Hanssen M, Bijl R, Vollebergh W. Prevalence of Psychotic Disorder and Cornrnunity Level of Psychotic Symptoms. Arch Gen Psychiatry. 2001;58:663-668.

47. Vicente B, Kohn R, Rioseco P, Saldivia S, Baker C, Torres S. Population prevalence of psychiatric disorders in Chile: 6-month and 1-month rates. Br J Psychiatry. 2004 Abr; 184:299-305.

48. Villaverde ML, Gracia R, de la Fuente J, González de Rivera JL, Rodríguez-Pulido F. Estudio comunitario de salud mental en población urbana de Tenerife. En: González de Rivera JL et al (eds): El método epidemiológico en salud mental. Barcelona, Masson-Salvat, 1993. Pags 187-214.

49. Waldo MC: Schizophrenia in Kosrae, Micronesia: prevalence, gender ratios, and clinical symptomatology. Schizophr Res 1999, 35:175-181.

50. Widerlöv B, Lindström E, von Knorring L. One-year prevalence of long-term functional psychosis in three different areas of Uppsala. Acta Psychiatr Scand. 1997 Dic;96(6):452-458.

51. Wittchen HU, Essau CA, von Zerssen D, Krieg JC, Zaudig M: Lifetime and six-month prevalence of mental disorders in the Munich FollowUp Study. Eur Arch Psychiatry Clin Neurosci 1992, 241:247-258.

52. Xiang Y, Ma X, Cai Z, Li S, Xiang Y, Guo H, et al. Prevalence and socio-demographic correlates of schizophrenia in Beijing, China. Schizophr. Res. 2008 Jul; 102(1-3):270-277.

53. Youssef HA, Kinsella A, Waddington JL: Evidence for geographical variations in the prevalence of schizophrenia in rural Ireland. Arch Gen Psychiatry 1991, 48:254-258. 